\title{
Detection of O-Linked-N-Acetylglucosamine Modification and Its Associated Enzymes in Human Degenerated Intervertebral Discs
}

\author{
Georgios Nikolaou ${ }^{1}$, Aristeidis H. Zibis ${ }^{1}$, Apostolos H. Fyllos ${ }^{1}$, Antonios Katsioulis ${ }^{2}$, \\ Sotirios Sotiriou ${ }^{3}$, Anastasios Kotrotsios ${ }^{1}$, Markos Sgantzos ${ }^{1}$, Aikaterini Vassiou ${ }^{1}$, Dimitrios L. Arvanitis ${ }^{1}$ \\ ${ }^{I}$ Department of Anatomy, Faculty of Medicine, University of Thessaly, Larissa, Greece \\ ${ }^{2}$ Department of Hygiene and Epidemiology, Faculty of Medicine, University of Thessaly, Larissa, Greece \\ ${ }^{3}$ Department of Embryology, Faculty of Medicine, University of Thessaly, Larissa, Greece
}

Study Design: Human herniated discs were obtained from discectomy specimens for the immunohistochemical detection of 0GIcNAc and O-GIcNAcase (OGA)/O-GIcNAc transferase (OGT).

Purpose: This study aimed to quantify the extent of 0-GICNAcylation and its associated enzymes (OGT/OGA) in human degenerated intervertebral discs.

Overview of Literature: The 0-GIcNAcylation of nuclear, cytoplasmic, and mitochondrial proteins as well as the effects of such post-translational modifications are currently the focus of extensive research. 0-GIcNAcylation is believed to contribute to the etiology of chronic illnesses by acting as a nutrient and stress sensor in the cellular environment. Mature intervertebral disc cells are chondrocyte-like cells, and 0-GIcNAc has been shown to promote chondrocyte apoptosis in vitro. We believe that 0-GIcNAcylation is a key regulator of disc degeneration.

Methods: Fifty-six specimens were fixed for 24 hours in a 10\% solution of neutral-buffered formaldehyde, dehydrated, and embedded in paraffin. Tissue slices (4- $\mu$ m-thick) were used for hematoxylin-eosin staining and immunohistochemistry.

Results: We found that 0-GIcNAcylation of cytoplasmic proteins was less than that of nuclear proteins in both single cells and cell clusters. Cytoplasmic 0-GIcNAcylation occurs subsequent to nuclear 0-GIcNAcylation and is directly proportional to disc degeneration. OGT and O-GICNAc expression levels were identical in all specimens examined.

Conclusions: 0-GICNAc and OGA/OGT expression is shown to correlate for the first time with intervertebral disc cell degeneration. Increasing disc degeneration is associated with increasing 0-GIcNAcylation in both nuclear and cytoplasmic proteins in human disc cells.

Keywords: Spine; Enzymes; Intervertebral disc degeneration; Immunohistochemistry

\section{Introduction}

The process of O-GlcNAcylation is defined as a post-trans- lational, bidirectional, dynamic modification of serine and threonine residues within nuclear, mitochondrial, and cytoplasmic proteins by O-linked $\beta$-N-acetylglucosamine

Received Mar 6, 2017; Revised Apr 4, 2017; Accepted Apr 10, 2017

Corresponding author: Aristeidis Hristos Zibis

Department of Anatomy, Faculty of Medicine, University of Thessaly, Panepistimiou 3, Viopolis, Larissa 41334, Greece

Tel: +30-6977228250, Fax: +30-2410289266, E-mail: ahzibis@gmail.com 
(O-GlcNAc). The O-GlcNAcylation substrate uridine diphospho-N-acetylglucosamine (UDP-GlcNAc) is directly produced by extracellular glucose metabolism via the hexosamine biosynthesis pathway, a major branch of the glycolytic pathway responsible for metabolizing $2 \%-5 \%$ of all cellular glucose [1]. As serum glucose levels rise in cases of diabetes or because of dietary uptake, more glucose is shunted into the hexosamine pathway, resulting in elevated levels of UDP-GlcNAc that can bind to proteins [2]. O-GlcNAcylation occurs post-translationally as a distinct form of glycosylation in both the nucleus and cytoplasm and is critical for cell transcription, proliferation, differentiation, and apoptosis. O-GlcNAcylation of serine and threonine residues is mediated by O-GlcNAc transferase (OGT) and reversed by O-GlcNAcase (OGA). The gene encoding OGT is highly conserved; its deletion is embryonically lethal $[3,4]$. O-GlcNAcylation is often suggested to serve as a nutrient sensor, as it is more common in hyperglycemic conditions, such as diabetes mellitus and metabolic syndrome $[5,6]$.

The roles of biochemical factors in intervertebral disc degeneration and pain have received increased focus recently. The human intervertebral disc contains chondrocyte-like cells within the nucleus pulposus and inner annulus fibrosus, and fibroblast-like cells are found in the outer annulus fibrosus. These cells are responsible for the synthesis and regulation of the disc matrix and control of homeostasis between extracellular matrix synthesis and degradation. Increased protein O-GlcNAcylation plays an important role in the stress response [7]. Disc cells are capable of producing pro-inflammatory mediators, such as tumor necrosis factor $\alpha$ (TNF- $\alpha$ ) and interleukin-1 (IL1), which may directly stimulate discogenic back pain by promoting neoangiogenesis and sensitizing peripheral nociceptors. Further matrix degradation triggered by these agents may result in pain by impairing mechanical stability or structural integrity [8].

The purpose of this study was to investigate the OGlcNAcylation of nuclear and cytoplasmic proteins in human degenerated intervertebral disc cells.

\section{Materials and Methods}

Fifty-six tissue samples were obtained from discectomy specimens of human herniated discs. The patient cohort included 30 men and 26 women, with a mean age of 45.6 years (range, 32 to 57 years). All patients presented with extruded nucleus pulposus and symptoms of severe radiculopathy. Specimens were fixed for 24 hours in a 10\% solution of neutral-buffered formaldehyde, dehydrated sequentially in $80 \%, 95 \%$, and $100 \%$ solutions of ethanol and $100 \%$ xylene, and embedded in paraffin. Slices $(4-\mu \mathrm{m}-$ thick) were used for hematoxylin-eosin staining and immunohistochemistry.

To identify O-GlcNAcylated proteins, we used the MAI-072 mouse monoclonal antibody RL2, which was obtained from Thermo Fisher Scientific (Waltham, MA, USA), at a 1:200 dilution, according to the manufacturer's instructions. To determine the localization of OGT, we used the PA5-13654 rabbit polyclonal antibody, which was obtained from Thermo Fisher Scientific, at a 1:100 dilution, according to the manufacturer's instructions. For the detection of OGA, we used the rabbit anti-MGEA5 antibody from Sigma-Aldrich Chemie GmbH (Taufkirchen, Germany). For immunohistochemistry experiments, the Genemed Kit Polymer HRP-containing goat antibody (South San Francisco, CA, USA) was used as a second antibody and for multicolor staining, according to the manufacturer's instructions.

Histological changes were graded according to the method described by Boos et al. [9]; we chose cell proliferation score (CPS) as a degeneration index. Immunohistochemistry grading was as follows: negative staining; weak staining $(1+)$; moderate staining $(2+)$; and intense staining (3+); for each intensity, if $>75 \%$ of the cell population was stained, was considered high, if $25 \%-75 \%$ of the cell population was stained, it was considered moderate, and if $<25 \%$ of the cell population was stained, it was considered low. All statistical analyses were performed using Microsoft Excel 2015 and SPSS ver. 21.0 (IBM Corp., Armonk, NY, USA).

The study was approved by the Institutional Review Board of University of Thessaly.

\section{Results}

The results of the immunohistochemistry experiments showed that there was nuclear and cytoplasmic staining of O-GlcNAc, OGT, and OGA in both single cells and cell clusters.

Staining results were identical for OGT and O-GlcNAcylated proteins. In all samples with a CPS of 5 , nuclear staining was intense (3+) in $>75 \%$ of single cells and cell clusters, whereas cytoplasmic staining was intense and 

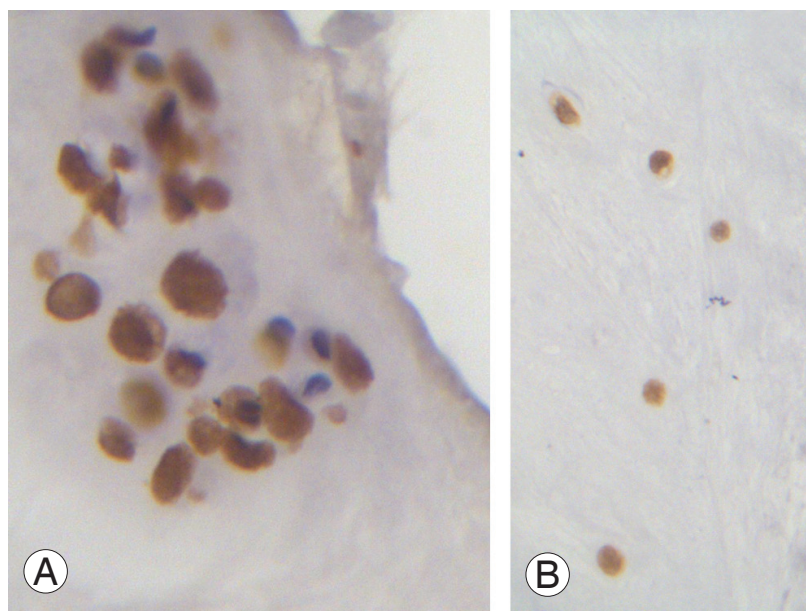

Fig. 1. (A) A large group of cells are shown. The overwhelming majority of cells shows intense nuclear and cytoplasmic staining (x400). (B) Single cells from an adjacent area of the same specimen show intense nuclear and cytoplasmic staining $(\times 400)$.
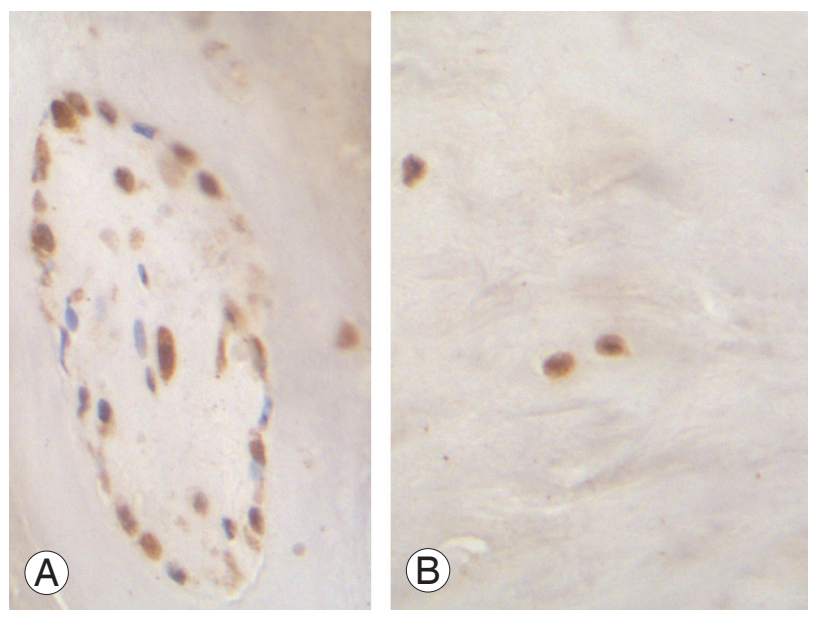

Fig. 2. (A) A group of cells stained for 0-GIcNAc transferase are shown. The great majority of cells shows intense nuclear and cytoplasmic staining. Seven cells remain unstained (blue nuclei) (×400). (B) Single cells from an adjacent area of the same specimen show intense nuclear and cytoplasmic staining $(\times 400)$.

high ( $>75 \%$ of cells) in $46 \%$ of samples and moderate $(2+)$ and high in the remaining samples. In all samples with a CPS of 4, nuclear staining was intense and high in single cells and cell clusters, whereas cytoplasmic staining was intense and high in only $16 \%$ of samples and moderate and high in the remaining samples. In samples with a CPS of 3, nuclear staining was intense and high in $77 \%$ of samples and moderate and high in the remaining four samples. Furthermore, in samples with a CPS of 3, cytoplasmic staining was moderate and high in 14 of 18 samples, and weak $(1+)$ and high in the remaining four

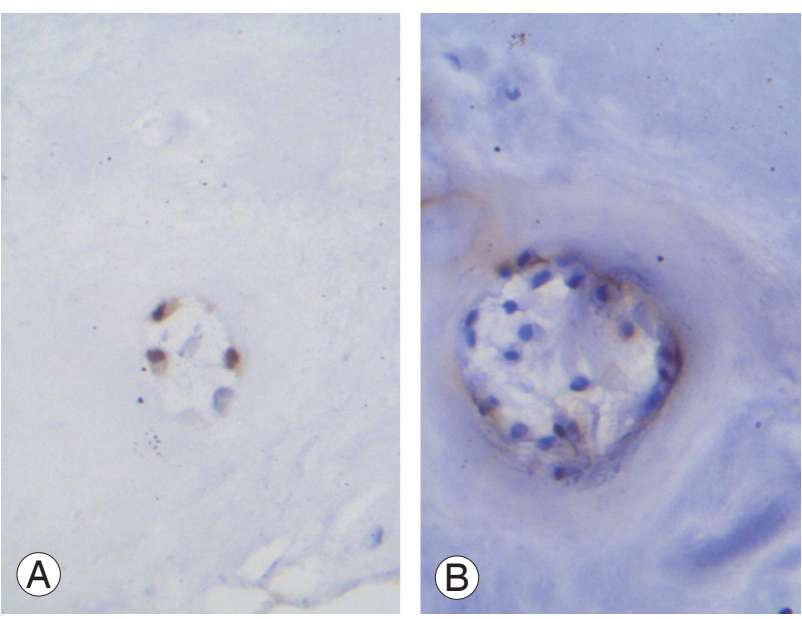

Fig. 3. (A) Immunohistochemistry for O-GIcNAcase (OGA) is shown. A group of three cells with intense nuclear and weak cytoplasmic staining are shown (x400). (B) A group of herniated intervertebral disc cells are shown. Immunohistochemistry for OGA is shown. All cells show negative nuclear staining (blue nuclei). Few cells with weak cytoplasmic staining (brown color) are shown $(\times 400)$.

samples.

Out of 26 samples with a CPS of 5, nuclear OGA staining was intense and high in $23 \%$ of samples, intense and moderate $(25 \%-75 \%$ of cells) in $23 \%$ of samples, weak and low ( $<25 \%$ of cells) in four samples, and completely negative in the remaining 10 samples. Cytoplasmic staining was weak and high in six samples, weak and moderate in six samples, and negative in 14 samples. In samples with a CPS of 4, both nuclear and cytoplasmic staining was negative in all 12 samples. In samples with a CPS of 3 , nuclear staining was intense and high in $11 \%$ of samples, intense and moderate in two samples, moderate and high in two samples, weak and low in two samples, and completely negative in the remaining 10 samples. Cytoplasmic staining was weak and high in $22 \%$ of samples, weak and moderate in two samples, and negative in the remaining 12 samples (Figs. 1-5).

Kendall's tau-b correlation coefficient was used to examine the relationship between the O-GlcNAc staining of nuclear and cytoplasmic proteins and CPS. A statistically significant moderate association was found between the O-GlcNAc staining of nuclear proteins and CPS (Kendall's coefficient $=0.334, p=0.027$ ), and a significantly positive strong association was found between the O-GlcNAc staining of cytoplasmic proteins and CPS (Kendall's coefficient $=0.513, p<0.001)$. Moreover, a significantly positive association was found between the O-GlcNAc staining of nuclear and cytoplasmic proteins (Kendall's coeffi- 


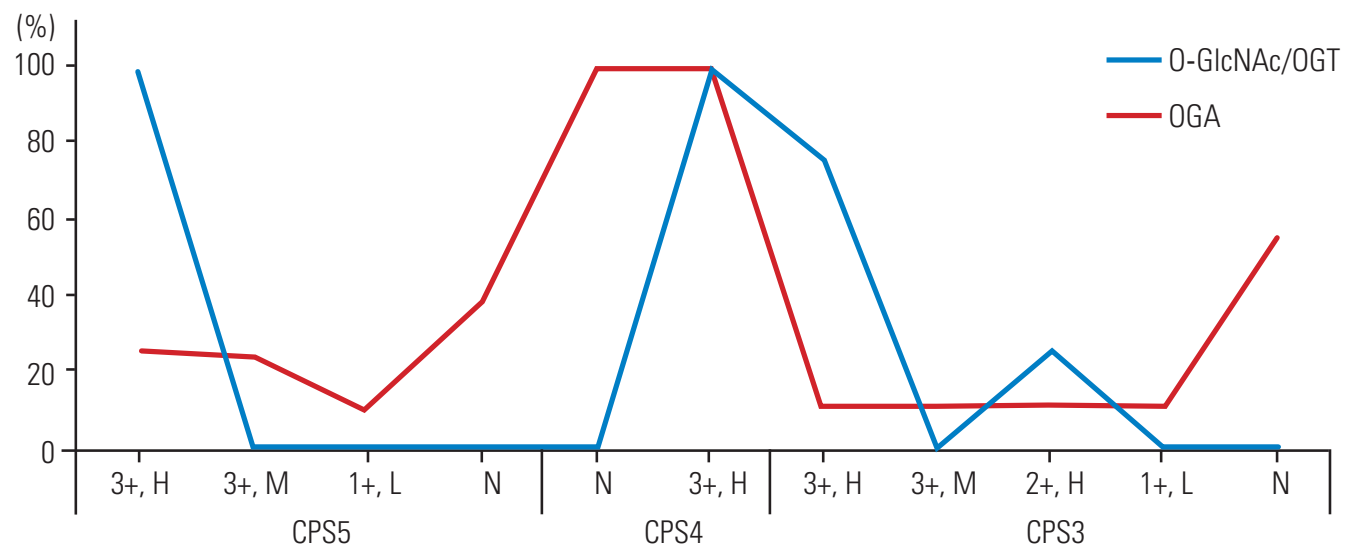

Fig. 4. Nuclear expression of O-GIcNAc/O-GIcNAc transferase (OGT) and O-GlcNAcase (OGA) is shown. CPS, cell proliferation score as defined int he text; $H$, high; $M$, moderate; $L$, low; $N$, none.

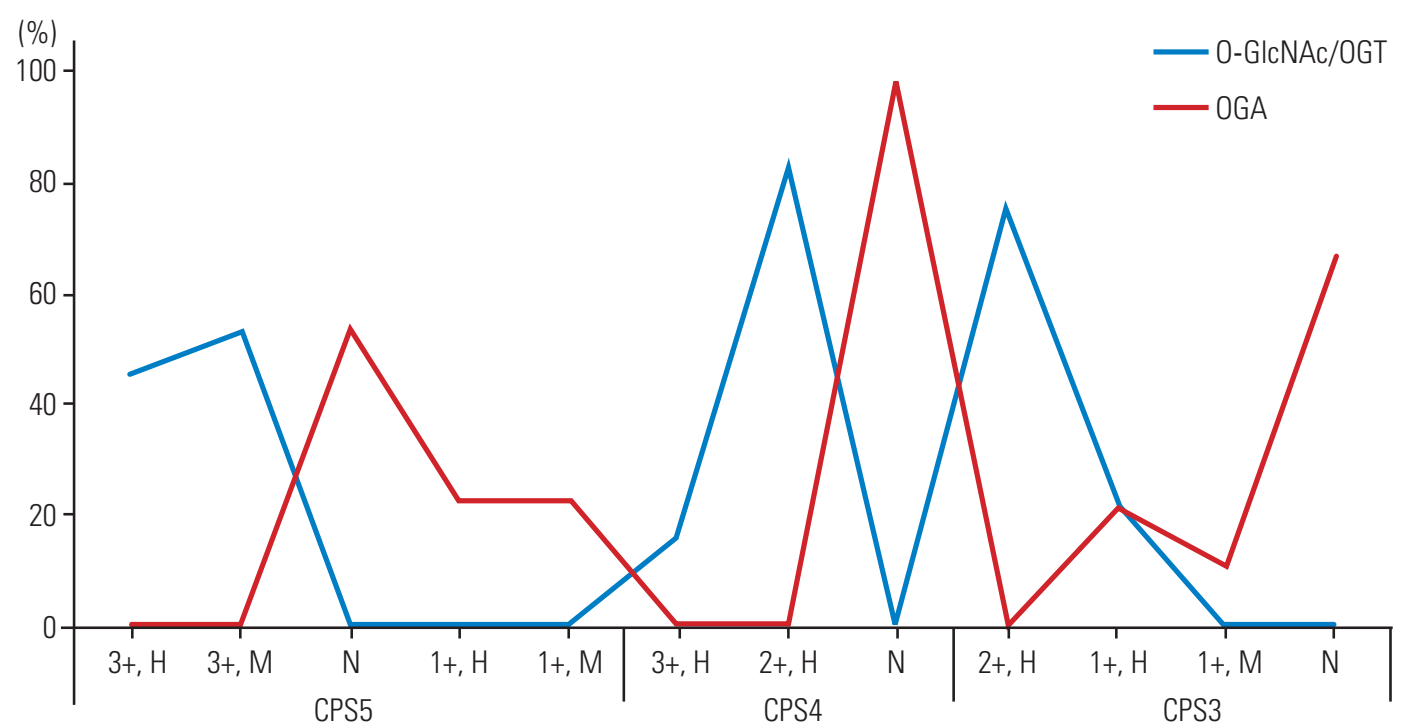

Fig. 5. Cytoplasmic expression of O-GIcNAc/O-GIcNAc transferase (OGT) and O-GIcNAcase (OGA) is shown. CPS, cell proliferation score as defined int he text; $\mathrm{H}$, high; $\mathrm{M}$, moderate; L, low; N, none.

cient $=0.530, p=0.025)$ with a low agreement $(25 \%)$.

\section{Discussion}

Intervertebral disc cells can be subjected to stress in the cellular environment due to avascularity and mechanical loading conditions. Such cells reside in a vulnerable environment characterized by hypoxia and low-glucose availability, as their nutrition is dependent upon diffusion from the neighboring capillary circulation $[10,11]$. Chondrocytes tend to proliferate and become hypertrophic under stress. Normal chondrocyte-like intervertebral disc cells exist in isolation, whereas degenerated disc cells are housed in stress-induced clusters that are derived from a single progenitor cell [12]. Stress often stimulates cellular glucose uptake, and increased protein O-GlcNAcylation has been shown to play an important role in this stress response [7], either through OGA inhibition or OGT overexpression. The elevation of O-GlcNAc levels before, or immediately after, the induction of cellular injury is protective in models of heat stress, oxidative stress, endoplasmic reticulum stress, hypoxia, ischemia reperfusion injury, and trauma hemorrhage $[13,14]$. Conversely, a sustained increase in O-GlcNAcylation contributes to the development of vascular and cardiac dysfunction [15]. O-GlcNAc represents not only a nutrient sensor but also a stress sensor. Cells can consequently modify O-GlcNAc levels to both respond and adapt to the cellular environ- 
ment. The purpose of the current study was to quantify $\mathrm{O}-\mathrm{GlcNAc}$ in human degenerated disc cells.

O-GlcNAc was present in both the nucleus and cytoplasm of degenerated herniated intervertebral disc cells at different levels, according to the degree of disc degeneration, which is reflected by the CPS. O-GlcNAc has a predominantly nuclear distribution, is associated with chromatin, and is found to bind with many cytoplasmic and mitochondrial proteins [16-18]. In all samples with a CPS of 4 or 5, nuclear staining was both intense and high. There were also no differences in the staining of single cells and cell clusters as a result of stress-induced proliferation. These findings suggest that either O-GlcNAc or stress similarly affect both single cells and cell clusters, or immunohistochemistry experiments are unable to resolve such subtle cellular differences. Nuclear staining was more intense in samples with a higher CPS in a statistically significant manner. The fact that nuclear staining was reduced in samples with a CPS of 3 compared with those with a higher CPS suggests that increased disc degeneration correlates with greater O-GlcNAcylation of nuclear proteins.

O-GlcNAc staining of cytoplasmic proteins was less than that of nuclear proteins. Cytoplasmic staining was intense and high in only $46 \%$ and $16 \%$ of samples with a CPS of 5 and 4, respectively. However, nuclear staining was intense in every sample with a CPS of 5 or 4 . No sample with a CPS of 3 had intense cytoplasmic staining, whereas $77 \%$ of such samples had intense nuclear staining. Furthermore, in samples with a CPS of 3, there was weak cytoplasmic staining in $23 \%$ of samples, compared with samples with a CPS of 5 or 4 . Therefore, cytoplasmic O-GlcNAcylation occurs subsequent to nuclear OGlcNAcylation and is directly proportional to disc degeneration.

OGT and O-GlcNAc expression levels were identical in all specimens examined. Identical increases in nuclear staining of these proteins were observed as intervertebral disc degeneration increased, with staining of cytoplasmic proteins less than that of nuclear proteins. However, a different pattern was observed for OGA expression. Negative OGA expression was observed in samples with a CPS of 4, whereas samples with a CPS of 3 or 5 displayed a range of OGA staining results. OGA cytoplasmic expression occurred subsequent to its nuclear expression. Therefore, OGA expression cannot be characterized as the opposite of O-GlcNAc or OGT expression, considering its negative expression in all samples with a CPS of 4 .

An example of an inflammatory role for a specific OGlcNAcylated protein is p65, a subunit of nuclear factor $\mathrm{kB}(\mathrm{NF}-\mathrm{kB})$, in rheumatoid arthritis (RA). Its effects were studied in both fibroblast-like synoviocytes (FLS) and a mouse model of collagen-induced arthritis (CIA). Cells were first treated with an OGA inhibitor and then with TNF- $\alpha$, resulting in the enhancement of O-GlcNAcylation. It was shown that hyper-O-GlcNAcylation increased both cell proliferation and expression levels of proinflammatory cytokines associated with RA in vitro and aggravated the severity of CIA in vivo. In contrast, hypoO-GlcNAcylation in response to OGA overexpression significantly attenuated inflammation in TNF- $\alpha$-stimulated FLS. O-GlcNAcylation of p65 enhanced inflammatory processes in TNF- $\alpha$-stimulated FLS via increases in the nuclear translocation of $\mathrm{p} 65$, protein-DNA binding, and transcriptional activity, which increased the expression levels of downstream genes [19]. In contrast, another study reported that glucosamine improved cardiac function in rats following hemorrhagic shock and attenuated the activation of NF-kB, which plays a central role in regulating the release of many cytokines, including TNF- $\alpha$ [20]. Finally, elevated O-GlcNAcylation appeared to significantly enhance colitis and colitis-associated colon cancer by modulating the DNA-binding activity of p65 in vivo [21].

It appears that hyperglycemia (in conditions of diabetes or dietary excess) has detrimental effects on human chondrocytes. Mature intervertebral disc cells are chondrocyte-like cells [22] that undergo the typical cascade of chondrogenic differentiation events (cell proliferation, extracellular matrix synthesis, cellular hypertrophy, matrix mineralization, vascular invasion, and eventually apoptosis), which allows cartilage remodeling into bone [23]. Diabetes can cause intervertebral disc degeneration by accelerating NP cell senescence and apoptosis [24]. Additionally, O-GlcNAc accumulation has been shown to increase chondrocyte apoptosis in vitro [25]. Furthermore, levels of O-GlcNAcylated proteins were found to increase four-fold in human osteoarthritic (OA) cartilage compared with healthy cartilage, a condition partly induced by IL-1 [26]. The increase in O-GlcNAcylation observed in OA cartilage was associated with a redistribution of expressed OGT isoforms, and an increase in OGA expression was also observed. Increased expression levels of OGT have been associated with O-GlcNAc accumulation 
in response to both acute stress and chronic damage [27]. In human OA chondrocytes, O-GlcNAc accumulation parallels increases in both OGT and OGA, suggesting that OGT and OGA levels may fluctuate in response to modifications of O-GlcNAc levels [25], similar to our results.

In recent research on diabetic rats, the apoptotic index of nucleus pulposus notochordal cells and the extent of transition of notochordal nucleus pulposus into pro-cartilaginous nucleus pulposus were significantly increased, confirming the hypothesis that hyperglycemia-induced $\mathrm{O}-\mathrm{GlcNAc}$ expression promotes and accelerates chondrocyte apoptosis, resulting in degeneration. Expression levels of matrix metalloproteinases-1, $-2,-3$, and -13 were also significantly increased in diabetic rats [28]. A higher incidence of degenerative disc disease in patients with diabetes and at a younger age than nondiabetic populations has been reported [29], suggesting that hyperglycemia contributes to the accelerated degeneration of intervertebral disc cells via O-GlcNAc.

This study's major strength is the use of immunohistochemistry to detect O-GlcNAc in degenerated intervertebral disc cells from human noncadaveric tissues, which has not been previously published. The major limitations of this study are the lack of similar immunohistochemistry data from normal discs and a lack of correlation between magnetic resonance imaging results for disc degeneration and our immunohistochemistry data.

\section{Conclusions}

O-GlcNAcylated proteins mediate numerous cellular processes. Research on the role of O-GlcNAc in intervertebral disc degeneration could elucidate the mechanism of intervertebral disc degeneration and lead to its prevention in various metabolic states.

\section{Conflict of Interest}

No potential conflict of interest relevant to this article was reported.

\section{ORCID}

Aristeidis Hristos Zibis:

https://orcid.org/0000-0001-7122-4317

Apostolos Haralampos Fyllos:

https://orcid.org/0000-0003-1384-2074
Antonios Katsioulis:

https://orcid.org/0000-0002-9337-7223

Markos Sgantzos: https://orcid.org/0000-0001-6337-4534

Dimitrios Leonidas Arvanitis:

https://orcid.org/0000-0001-6435-7461

\section{References}

1. Chatham JC, Not LG, Fulop N, Marchase RB. Hexosamine biosynthesis and protein $\mathrm{O}$-glycosylation: the first line of defense against stress, ischemia, and trauma. Shock 2008;29:431-40.

2. Ma J, Hart GW. Protein O-GlcNAcylation in diabetes and diabetic complications. Expert Rev Proteomics 2013;10:365-80.

3. Lubas WA, Frank DW, Krause M, Hanover JA. OLinked GlcNAc transferase is a conserved nucleocytoplasmic protein containing tetratricopeptide repeats. J Biol Chem 1997;272:9316-24.

4. Shafi R, Iyer SP, Ellies LG, et al. The O-GlcNAc transferase gene resides on the $\mathrm{X}$ chromosome and is essential for embryonic stem cell viability and mouse ontogeny. Proc Natl Acad Sci U S A 2000;97:5735-9.

5. Hart GW, Housley MP, Slawson C. Cycling of Olinked beta- $\mathrm{N}$-acetylglucosamine on nucleocytoplasmic proteins. Nature 2007;446:1017-22.

6. Olivier-Van Stichelen S, Hanover JA. You are what you eat: O-linked $\mathrm{N}$-acetylglucosamine in disease, development and epigenetics. Curr Opin Clin Nutr Metab Care 2015;18:339-45.

7. Zachara NE, Hart GW. O-GlcNAc a sensor of cellular state: the role of nucleocytoplasmic glycosylation in modulating cellular function in response to nutrition and stress. Biochim Biophys Acta 2004;1673:13-28.

8. Risbud MV, Shapiro IM. Role of cytokines in intervertebral disc degeneration: pain and disc content. Nat Rev Rheumatol 2014;10:44-56.

9. Boos N, Weissbach S, Rohrbach H, Weiler C, Spratt KF, Nerlich AG. Classification of age-related changes in lumbar intervertebral discs: 2002 Volvo Award in basic science. Spine (Phila Pa 1976) 2002;27:2631-44.

10. Rajasekaran S, Babu JN, Arun R, Armstrong BR, Shetty AP, Murugan S. ISSLS prize winner: A study of diffusion in human lumbar discs: a serial magnetic resonance imaging study documenting the influence of the endplate on diffusion in normal and degenerate discs. Spine (Phila Pa 1976) 2004;29:2654-67. 
11. Grunhagen T, Shirazi-Adl A, Fairbank JC, Urban JP. Intervertebral disk nutrition: a review of factors influencing concentrations of nutrients and metabolites. Orthop Clin North Am 2011;42:465-77.

12. Sharp CA, Roberts S, Evans H, Brown SJ. Disc cell clusters in pathological human intervertebral discs are associated with increased stress protein immunostaining. Eur Spine J 2009;18:1587-94.

13. Zachara NE, Molina H, Wong KY, Pandey A, Hart GW. The dynamic stress-induced "O-GlcNAc-ome" highlights functions for $\mathrm{O}-\mathrm{GlcNAc}$ in regulating DNA damage/repair and other cellular pathways. Amino Acids 2011;40:793-808.

14. Arvanitis LD, Vassiou K, Kotrotsios A, Sgantzos MN. Hypoxia upregulates the expression of the O-linked $\mathrm{N}$-acetylglucosamine containing epitope $\mathrm{H}$ in human ependymal cells. Pathol Res Pract 2011;207:91-6.

15. Dassanayaka S, Jones SP. O-GlcNAc and the cardiovascular system. Pharmacol Ther 2014;142:62-71.

16. Kelly WG, Hart GW. Glycosylation of chromosomal proteins: localization of O-linked $\mathrm{N}$-acetylglucosamine in Drosophila chromatin. Cell 1989;57:243-51.

17. Love DC, Kochan J, Cathey RL, Shin SH, Hanover JA. Mitochondrial and nucleocytoplasmic targeting of $\mathrm{O}$ linked GlcNAc transferase. J Cell Sci 2003;116:64754.

18. Arvanitis DL, Arvanitis LD, Panourias IG, Kitsoulis P, Kanavaros P. Mitochondria-rich normal, metaplastic, and neoplastic cells show overexpression of the epitope $\mathrm{H}$ recognized by the monoclonal antibody $\mathrm{H}$. Pathol Res Pract 2005;201:319-24.

19. Kim HB, Lee SW, Mun CH, et al. O-linked Nacetylglucosamine glycosylation of p65 aggravated the inflammation in both fibroblast-like synoviocytes stimulated by tumor necrosis factor-alpha and mice with collagen induced arthritis. Arthritis Res Ther 2015;17:248.

20. Zou L, Yang S, Champattanachai V, et al. Glucos- amine improves cardiac function following traumahemorrhage by increased protein O-GlcNAcylation and attenuation of NF- $\kappa \mathrm{B}$ signaling. Am J Physiol Heart Circ Physiol 2009;296:H515-23.

21. Yang YR, Kim DH, Seo YK, et al. Elevated OGlcNAcylation promotes colonic inflammation and tumorigenesis by modulating NF- $\kappa \mathrm{B}$ signaling. Oncotarget 2015;6:12529-42.

22. Trout JJ, Buckwalter JA, Moore KC. Ultrastructure of the human intervertebral disc: II. Cells of the nucleus pulposus. Anat Rec 1982;204:307-14.

23. Kronenberg HM. Developmental regulation of the growth plate. Nature 2003;423:332-6.

24. Jiang L, Zhang X, Zheng X, et al. Apoptosis, senescence, and autophagy in rat nucleus pulposus cells: implications for diabetic intervertebral disc degeneration. J Orthop Res 2013;31:692-702.

25. Andres-Bergos J, Tardio L, Larranaga-Vera A, Gomez $\mathrm{R}$, Herrero-Beaumont G, Largo $\mathrm{R}$. The increase in $\mathrm{O}$-linked $\mathrm{N}$-acetylglucosamine protein modification stimulates chondrogenic differentiation both in vitro and in vivo. J Biol Chem 2012;287:33615-28.

26. Tardio L, Andres-Bergos J, Zachara NE, et al. Olinked $\mathrm{N}$-acetylglucosamine $(\mathrm{O}-\mathrm{GlcNAc}$ ) protein modification is increased in the cartilage of patients with knee osteoarthritis. Osteoarthritis Cartilage 2014;22:259-63.

27. Zachara NE. The roles of O-linked beta-N-acetylglucosamine in cardiovascular physiology and disease. Am J Physiol Heart Circ Physiol 2012;302:H1905-18.

28. Won HY, Park JB, Park EY, Riew KD. Effect of hyperglycemia on apoptosis of notochordal cells and intervertebral disc degeneration in diabetic rats. J Neurosurg Spine 2009;11:741-8.

29. Sakellaridis N. The influence of diabetes mellitus on lumbar intervertebral disk herniation. Surg Neurol 2006;66:152-4. 